\title{
Eclipsing binaries in the Galactic bulge: candidates for distance estimates ${ }^{\star}, \star \star$
}

\author{
M. A. T. Groenewegen
}

\author{
Instituut voor Sterrenkunde, K.U. Leuven, PACS-ICC, Celestijnenlaan 200B, 3001 Leuven, Belgium \\ e-mail: groen@ster.kuleuven.be
}

Received 16 February 2005 / Accepted 26 April 2005

\begin{abstract}
The 222000 I-band light curves of variable stars detected by the OGLE-II survey in the direction of the Galactic Bulge have been searched for eclipsing binaries (EBs). A previously developed code to analyze lightcurve shapes and identify long period variables (LPVs) has been adapted to identify EBs. The parameters in the modified code have been optimised to recover a list of about 140 detached EBs in the Small Magellanic Cloud previously identified in the literature as particularly well suited for distance estimates (and wich have periods $\gtrsim 0.85$ days). The power of the code is demonstrated by identifying 16 and 178 previously uncatalogued EBs in the SMC and LMC, respectively. Among the 222000 variable stars in the direction of the Galactic Bulge 3053 EBs have been identified. Periods and phased lightcurves are presented.
\end{abstract}

Key words. stars: distances - binaries: eclipsing - Galaxy: center

\section{Introduction}

Detached double-lined eclipsing binaries (hereafter EBs for short) are very suitable as primary distance indicators when accurate photometry and radial velocity (RV) data are combined (e.g. Andersen 1991). The massive photometric monitoring data obtained in the course of the micro lensing surveys in the 1990's of the Small and Large Magellanic Clouds has revealed many candidate detached eclipsing binaries based on the shape of the lightcurves (Grison et al. 1995; Alcock et al. 1997; Udalski et al. 1998; Bayne et al. 2002; Wyrzykowski et al. 2003, 2004).

Some of them have been followed-up with spectroscopy to obtain RV data and perform a combined lightcurve and $\mathrm{RV}$ curve analysis to obtain the distance to the system (see e.g., Guinan et al. 1998; Hilditch et al. 2005; and the review by Clausen 2004, for a list of systems with recent distance estimates). What makes EBs particularly powerful is that the error in the distance estimate in a single well-observed system (0.06-0.10 in distance modulus, see Clausen 2004) is already comparable to the dispersion in optical and infrared Cepheid $P L$-relation based on hundreds of stars (e.g. Sandage et al. 2004; Nikolaev et al. 2003).

In the present paper a list of eclipsing binaries in the direction of the Galactic Bulge (GB) is presented based on analysis

^ Full Tables 2 and B.1 are only available in electronic form at the CDS via anonymous ftp to

cdsarc.u-strasbg.fr $(130.79 .128 .5)$ or via

http://cdsweb.u-strasbg.fr/cgi-bin/qcat?J/A+A/439/559

$\star \star$ Appendices A, B and full Fig. 1 are only available in electronic form at http://www. edpsciences.org of OGLE-II data. Previously, 1650 EBs have been identified in OGLE-I data (Udalski et al. 1994, 1995a,b, 1996, 1997).

The paper is structured as follows. In Sect. 2 the OGLE-II surveys is briefly described. In Sect. 3 the model to identify EBs from the lightcurve shape is briefly presented. The results are presented in Sect. 4, and discussed in Sect. 5.

\section{The data sets}

The OGLE-II micro lensing experiment observed fourty-nine fields in the direction of the GB. Each field has a size $14.2^{\prime} \times$ $57^{\prime}$ and was observed in $B V I$, with an absolute photometric accuracy of 0.01-0.02 mag (Udalski et al. 2002). Table 1 lists the galactic coordinates of the field centers and the total number of sources detected in these fields.

Wozniak et al. (2002) present a catalog of about 222000 variable objects based on the OGLE observations covering 1997-1999, applying the difference image analysis (DIA) technique on the $I$-band data. The data files containing the $I$-band data of the candidate variable stars was downloaded from the OGLE homepage (http:// sirius. astrouw . edu.pl/ ogle/).

\section{Analysis of the lightcurve shape and selecting eclipsing binaries}

The model to analyse the lightcurve shape and identify long period variables (LPVs) is described in detail in Appendices A-C in Groenewegen (2004, herafter G04).

Briefly, a first code (see for details Appendix A in G04) sequentially reads in the I-band data for the objects, 
Table 1. Properties of the OGLE-fields and the number of detected eclipsing binaries.

\begin{tabular}{|c|c|c|c|c|c|c|}
\hline BUL_SC & $\bar{l}$ & $\overline{c b}$ & $\overline{\text { Total }}^{a}$ & Variable $^{b}$ & 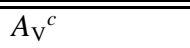 & $\overline{\mathrm{EBs}^{d}}$ \\
\hline 1 & 1.08 & -3.62 & 730 & 4597 & $1.68 / 1.49$ & 77 \\
\hline 2 & 2.23 & -3.46 & 803 & 5279 & $1.55 / 1.65$ & 70 \\
\hline 3 & 0.11 & -1.93 & 806 & 8393 & 2.89 & 130 \\
\hline 4 & 0.43 & -2.01 & 774 & 9096 & $2.59 / 2.94$ & 146 \\
\hline 5 & -0.23 & -1.33 & 434 & 7257 & $5.73 /-/ 4.13$ & 39 \\
\hline 6 & -0.25 & -5.70 & 514 & 3211 & 1.37 & 41 \\
\hline 7 & -0.14 & -5.91 & 463 & 1618 & $1.33 / 1.28$ & 21 \\
\hline 8 & 10.48 & -3.78 & 402 & 2331 & 2.14 & 29 \\
\hline 9 & 10.59 & -3.98 & 330 & 1847 & 2.08 & 18 \\
\hline 10 & 9.64 & -3.44 & 458 & 2499 & 2.23 & 36 \\
\hline 11 & 9.74 & -3.64 & 426 & 2256 & 2.27 & 25 \\
\hline 12 & 7.80 & -3.37 & 535 & 3476 & $2.29 / 2.20$ & 36 \\
\hline 13 & 7.91 & -3.58 & 570 & 3084 & $2.06 / 1.82$ & 41 \\
\hline 14 & 5.23 & 2.81 & 619 & 4051 & 2.49 & 83 \\
\hline 15 & 5.38 & 2.63 & 601 & 3853 & 2.77 & 41 \\
\hline 16 & 5.10 & -3.29 & 700 & 4802 & $2.15 / 2.23$ & 48 \\
\hline 17 & 5.28 & -3.45 & 687 & 4690 & $1.94 / 2.29$ & 46 \\
\hline 18 & 3.97 & -3.14 & 749 & 5805 & 1.83 & 78 \\
\hline 19 & 4.08 & -3.35 & 732 & 5255 & 2.74 & 61 \\
\hline 20 & 1.68 & -2.47 & 785 & 5910 & $1.94 / 2.02$ & 119 \\
\hline 21 & 1.80 & -2.66 & 883 & 7449 & $1.83 / 1.78$ & 116 \\
\hline 22 & -0.26 & -2.95 & 715 & 5589 & 2.74 & 91 \\
\hline 23 & -0.50 & -3.36 & 723 & 4815 & 2.70 & 68 \\
\hline 24 & -2.44 & -3.36 & 612 & 4304 & 2.52 & 68 \\
\hline 25 & -2.32 & -3.56 & 622 & 3046 & 2.34 & 77 \\
\hline 26 & -4.90 & -3.37 & 728 & 4713 & 1.86 & 76 \\
\hline 27 & -4.92 & -3.65 & 691 & 3691 & 1.69 & 66 \\
\hline 28 & -6.76 & -4.43 & 406 & 1472 & 1.64 & 26 \\
\hline 29 & -6.64 & -4.62 & 492 & 2398 & 1.53 & 44 \\
\hline 30 & 1.94 & -2.84 & 762 & 6893 & $1.91 / 1.78$ & 137 \\
\hline 31 & 2.23 & -2.94 & 790 & 4789 & $1.81 / 1.74$ & 87 \\
\hline 32 & 2.34 & -3.14 & 797 & 5007 & $1.61 / 1.82$ & 99 \\
\hline 33 & 2.35 & -3.66 & 739 & 4590 & $1.70 / 1.82$ & 44 \\
\hline 34 & 1.35 & -2.40 & 961 & 7953 & $2.52 / 2.32$ & 127 \\
\hline 35 & 3.05 & -3.00 & 771 & 5169 & $1.84 / 2.20$ & 64 \\
\hline 36 & 3.16 & -3.20 & 873 & 8805 & $1.62 / 1.52$ & 85 \\
\hline 37 & 0.00 & -1.74 & 664 & 8367 & 3.77 & 119 \\
\hline 38 & 0.97 & -3.42 & 710 & 5072 & $1.83 / 1.94$ & 119 \\
\hline 39 & 0.53 & -2.21 & 784 & 7338 & $2.63 / 2.70$ & 144 \\
\hline 40 & -2.99 & -3.14 & 631 & 4079 & 2.94 & 48 \\
\hline 41 & -2.78 & -3.27 & 603 & 4035 & 2.65 & 55 \\
\hline 42 & 4.48 & -3.38 & 601 & 4360 & 2.29 & 67 \\
\hline 43 & 0.37 & 2.95 & 474 & 3351 & 3.67 & 34 \\
\hline 44 & -0.43 & -1.19 & 319 & 7836 & $6.0 /-/ 6.00$ & 32 \\
\hline 45 & 0.98 & -3.94 & 627 & 2262 & $1.64 / 1.53$ & 4 \\
\hline 46 & 1.09 & -4.14 & 552 & 2057 & $1.71 / 1.65$ & 3 \\
\hline 47 & -11.19 & -2.60 & 301 & 1152 & 2.60 & 7 \\
\hline 48 & -11.07 & -2.78 & 287 & 973 & 2.35 & 9 \\
\hline 49 & -11.36 & -3.25 & 251 & 826 & 2.09 & 6 \\
\hline Total & & & 30490 & 221701 & & 3053 \\
\hline
\end{tabular}

${ }^{a}$ Total number of objects detected in the field. From Udalski et al. (2002), in units of $10^{3}$ objects.

${ }^{b}$ Total number of candidate variable stars. From Wozniak et al. (2002).

${ }^{c}$ Visual extinction. From Sumi (2004), except for SC44, where $A_{\mathrm{V}}=6.0$ has been adopted based on the proximity to C5. The second value when listed - comes from Popowski et al. (2003). The third value - when listed - comes from Schultheis et al. (1999).

${ }^{d}$ Total number of EBs. 
determines periods through Fourier analysis, fits sine and cosine functions to the light curve through linear least-squares fitting and makes the final correlation with the pre-prepared DENIS and 2MASS source lists. All the relevant output quantities are written to file. This part of the code is adapted significantly to better deal with the specific properties of eclipsing binaries w.r.t. pulsational variables, as described in Appendix A of the present paper. Brief, phase dispersion minimization is used next to Fourier analysis to select the true orbital period, and the EBs candidates are selected based on statistical properties of the phased light curve.

The output file of the first code is read in by the second code (see for details Appendix B in G04). A further selection may be applied (typically on period, amplitude and mean I-magnitude), multiple entries are filtered out (i.e. objects that appear in different OGLE fields), and a correlation is made with pre-prepared lists of objects for cross-identification. The output of the second code is a list with EB candidates.

The third step (for details see Appendix C in G04) consists of a visual inspection of the fits to the (phased) light curves of the candidate EBs and a literature study through a correlation with the SIMBAD database. Non-EBs are removed, and sometimes the fitting is redone. The final list of EB candidates is compiled.

\section{Results}

\subsection{Testing the code on selected SMC data}

In order to set and finetune the parameters described in Appendix A that define the selection of an EB, and also to demonstrate that with these particular settings EBs can indeed be retrieved, the code is tested and run on the OGLE data of variable stars in the Magellanic Clouds (hereafter MCs; Zebrun et al. 2001).

As the ultimate aim of the project is to define EBs in the GB suitable for distance determinations the parameters that define the selection of EBs ( 3 statistical parameters that define the shape of the phased lightcurve, and the parameters significance and hifac of the NUMERICAL RECIPES (Press et al. 1992) subroutine fasper) are tuned to retrieve the stars in the lists of "ideal" distance indicators in the SMC previously selected by Udalski et al. (1998) and Wyithe \& Wilson (2001) based on the first release of OGLE data (based on a shorter timespan of OGLE data and aperture photometry instead of DIA). What constitutes an "ideal" EB in terms of a distance indicator is difficult to quantify exactly. Typically the system should be well-detached with small values of the stellar radii relative to the semi-major axis $(r / a \lesssim 0.2)$ and have deep eclipses (e.g. Wyithe \& Wilson 2001).

Not all of the stars from Udalski et al. (1998) and Wyithe $\&$ Wilson (2001) are listed in the Zebruń et al. (2001) data set. In fact, Wyrzykowski et al. (2004) state that of the 1527 EBs listed by Udalski et al. (1998) only 935 can be found in the DIA catalog of variable stars by Zebruń et al. (2001), and quote the incompleteness of the DIA catalog for faint stars as the probable reason. Of the 153 stars listed by Udalksi et al. (1998) and the 22 unique additional objects in Tables 4 and 5 of
Wyithe \& Wilson (i.e. not already listed in Udalski et al.), 142 are found back by Zebruń et al. (2001), and these stars represent the test data set.

Of these 142, 137 are correctly identified as EBs by the program using the parameters mentioned in Appendix A. One object (OGLE005102.88-730941.0) is found with the period as listed by Udalski et al. (1998) but the phased light curve is poor with the DIA data set, and the object is not suitable as a distance indicator from the present data. Two stars would have been correctly identified as EBs were it not that the significance of the peak of the Fourier spectrum is above the adopted threshold. The other two are missed because the parameters that are being used to describe the shape of the phased lightcurve are outliers. At the end of the day, it is deemed acceptable to miss 4 stars out of a 141 suitable candidates. It should be mentioned that these "ideal" distance indicators have orbital periods larger than 0.85 days and so the code is optimised to be sensitive to this period range, and hence will be biased against shorter orbital periods.

\subsection{Applying the code to $S M C$ and $L M C$ data}

As a further test, the code is applied to the full set of 68000 lightcurves of variable stars in the SMC and LMC by Zebrun et al. (2001), and compared to the outcome of the previous work by Wyrzykowski et al. (2003, 2004), based on the same dataset as is the present work.

Wyrzykowski et al. (2003) find $2580 \mathrm{EBs}$ in the LMC, and Wyrzykowski et al. (2004) find 1351 EBs in the SMC, based on an artificial neural network. Note that they attempted to find all types of eclipsing binaries, while the aim of the present paper is to detect EBs potentially suitable for distance determination, hence preferentially detached systems.

Applying the code resulted in 1856 LMC and 752 SMC candidate EBs.

For the SMC, 714 of the 752 objects are listed in Wyrzykowski et al. (2004) as EBs. Of the 38 not listed in Wyrzykowski et al., 20 are eliminated at the visual inspection stage as the phased lightcurves are not typical of that of an EB; 13 are in fact known Cepheids. Of the 18 remaining stars, 2 are classified as EB by MOA (Bayne et al. 2002), and 16 are new EBs not previously identified. Names, periods and the phased lightcurve are presented in Appendix B.

For the LMC, 1616 of the 1856 objects are listed in Wyrzykowski et al. (2003) as EBs. Of the 240 not listed in Wyrzykowski et al., 51 are eliminated at the visual inspection stage as the phased lightcurves are not typical of that of an EB; 7 are known Cepheids, 2 are known RR Lyrae and 24 are LPVs. Of the 189 remaining stars, 11 are classified as EB by MACHO (Alcock et al. 1997), and 178 are new EBs not previously identified. Names, periods and the phased lightcurve are presented in Appendix B.

The conclusion is that the simple method developed for detecting EBs is complementary to the neural network method used in Wyrzykowski et al. $(2003,2004)$ as, considering both MCs, about $7.5 \%$ of the detected objects are newly 

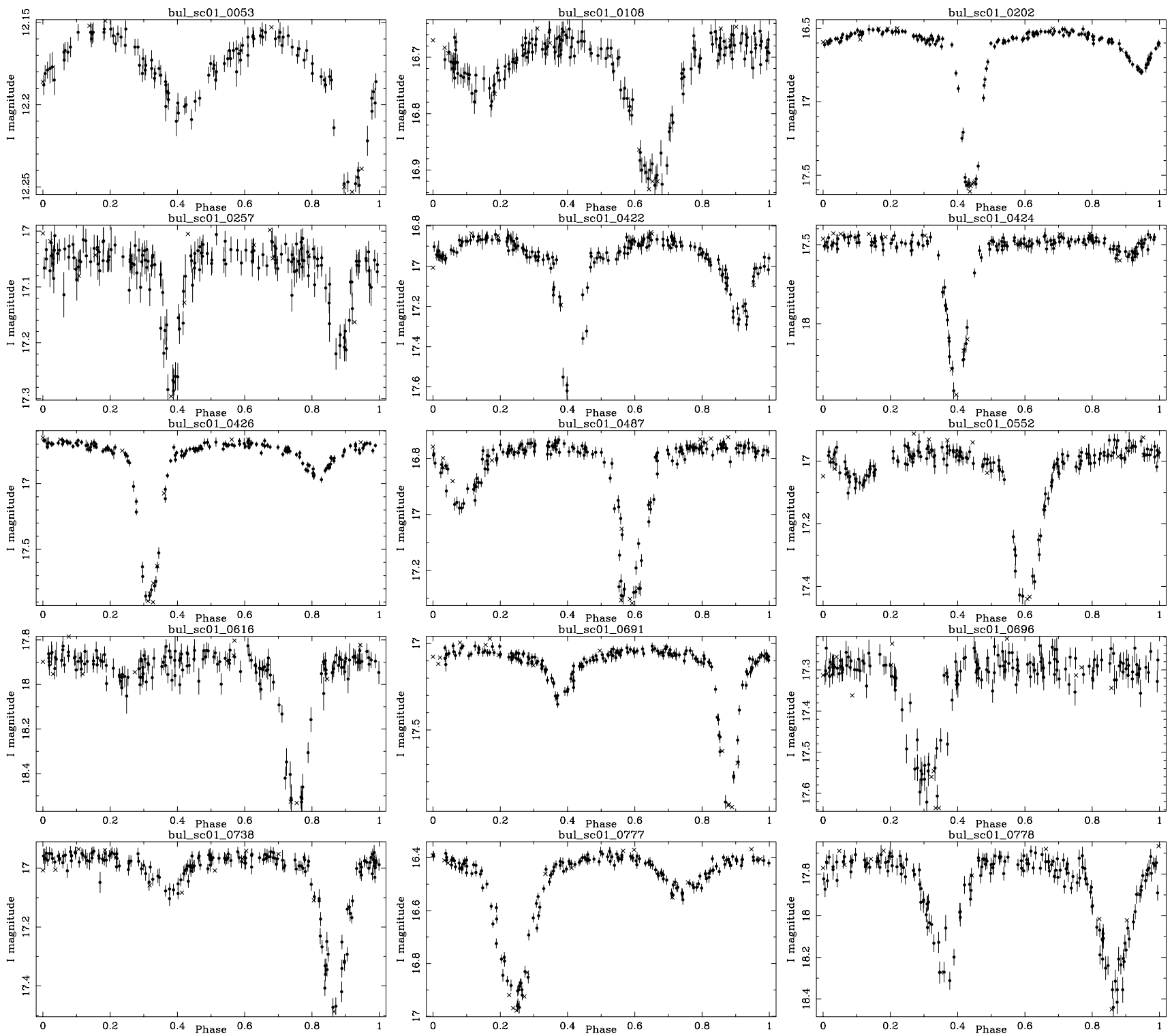

Fig. 1. First 15 entries of the electronically available figure with all phased lightcurves of eclipsing binaries in the direction of the Galactic Bulge. Crosses indicate data points not included in the analysis. Phase zero is given by the Julian Date listed in Table 2.

discovered EBs. The number of false candidates (mostly cepheids, RR Lyrae and LPVs) is about $2.5 \%$.

\subsection{Applying the code to the Galactic Bulge}

The numerical code is applied to the OGLE-II data in the direction of the Galactic Bulge. After visual inspection of the lightcurves a sample of 3053 objects remain. The largest number of objects that are removed at this stage are LPVs especially at longer periods ( $P_{\text {binary }} \gtrsim 150$, respectively $P_{\text {pulsation }} \gtrsim$ 75 days) and EBs with orbital periods $\lesssim 0.85$ days where an alias frequency is picked up, which nevertheless phases well enough to fulfill the initial selection criteria.

The number of objects per field is listed in the last column of Table 1 . Table 2 lists the 3053 objects with periods, and Fig. 1 displays the phased lightcurves. The typical error in the period is $3 \times 10^{-4} P$. Only one object is listed in the SIMBAD database, namely bul_sc26_3510 (RA = $17 \mathrm{~h} 47 \mathrm{~m} 28.25 \mathrm{~s}$, Dec $=-34 \mathrm{~d} 46 \mathrm{~m} 31.0 \mathrm{~s}$ ), a.k.a. MM5-A V47 in Udalski et al. (1997) and classified as a W UMa-type binary. The period quoted there is $1.10577 \mathrm{~d}$ while in the present analysis $1.105801 \mathrm{~d}$ is derived.

\section{Summary}

The paper presents a simple method to detect eclipsing binaries based on the shape of the phased lightcurve. In view of the ultimate aim to use EBs to derive the distance to the Galactic Centre, the parameters of the model are finetuned to retrieve a large sample of EBs in the SMC previously classified in the literature as potentially suitable for distance determinations. The code is run on the 68000 OGLE variable stars in LMC and SMC to find 194 apparently previously unclassified EBs, and on the about 222000 OGLE variable stars in the direction of the Bulge 
Table 2. First entries in the electronically available table, which lists: OGLE-name, oribital period, and time used to define phase zero.

\begin{tabular}{ccc}
\hline \hline OGLE name & Period $(d)$ & $T^{a}$ \\
\hline bul_sc01_0053 & 2.521648 & 530.819 \\
bul_sc01_0108 & 1.532373 & 550.789 \\
bul_sc01_0202 & 4.513265 & 535.895 \\
bul_sc01_0257 & 2.378940 & 540.765 \\
bul_sc01_0422 & 4.201144 & 530.836 \\
bul_sc01_0424 & 1.738786 & 530.836 \\
bul_sc01_0426 & 4.236257 & 530.819 \\
bul_sc01_0487 & 1.782421 & 550.789 \\
bul_sc01_0552 & 2.049995 & 550.789 \\
bul_sc01_0616 & 1.761581 & 530.837 \\
bul_sc01_0691 & 3.350188 & 535.895 \\
bul_sc01_0696 & 1.329869 & 550.789 \\
bul_sc01_0738 & 1.427248 & 540.765 \\
bul_sc01_0777 & 1.578524 & 550.789 \\
bul_sc01_0778 & 1.107325 & 540.765 \\
bul_sc01_0851 & 8.904982 & 550.789 \\
\hline
\end{tabular}

${ }^{a} T=$ (JD-2 450000$)$ used to define zero phase in Fig. 1.

First entries only. Complete table available in electronic form at the CDS

to find about $3000 \mathrm{EBs}$. It should be stressed that due to the finetuning of the model no short period EBs $(P \lesssim 0.85 \mathrm{~d})$ are among these.

It should also be stressed that altough the code was tuned to retrieve EBs peviously classified in the literature as suitable for distance determinations, the converse is not necesarily true: the objects returned by the code and listed in Tables 2 and B. 1 are not automatically suitable as potential distance indicators. For example, none of the 15 objects displayed in Fig. B.1 seems suitable for this purpose. In this case this is not surprising as only newly discovered EBs, not in the exhaustive lists of Wyrzykowski et al. (2003, 2004), are listed and so these objects have relatively low $\mathrm{S} / \mathrm{N}$ lightcurves. Of the lightcurves displayed in Fig. 1 only a few appear suitable for follow-up studies.

In the future it is planned to select a small sub-sample among the 3000 objects based on a proper analysis of the lightcurve in terms of radii determination and eclipse depth and obtain multi-epoch spectroscopic data in order to derive the fundamental parameters (mass, radius, effective temperature, metallicity) and distance.

Acknowledgements. This research has made use of the SIMBAD database, operated at CDS, Strasbourg, France.

\section{References}

Alcock, C., Allsman, R. A., Alves, D., et al. 1997, AJ, 114, 326

Andersen, J. 1991, A\&AR, 3, 91

Bayne, G., Tobin, W., Pritchard, J. D., et al. 2002, MNRAS, 331, 609

Clausen, J. V. 2004, NewAR, 48, 679

Grison, P., Beaulieu, J.-P., Pritchard, J. D., et al. 1995, A\&AS, 109, 447

Groenewegen, M. A. T. 2004, A\&A, 425, 595 (G04)

Groenewegen, M. A. T., \& Blommaert, J. A. D. L. 2005, A\&A, submitted

Guinan, E. F., Fitzpatrick, E. L., DeWarf, L. E., et al. 1998, ApJ, 509, L21

Hilditch, R. W., Howarth, I. D., \& Harries, T. J. 2005, MNRAS, 357, 304

Nikolaev, S., Drake, A. J., Keller, S. C., et al. 2003, ApJ, 601, 260

Popowski, P., Cook, K. H., \& Becker, A. C. 2003, AJ, 126, 2910

Press, W. H., Teukolsky, S. A., Vetterling, W. T., \& Flannery, B. P. 1992, Numerical Recipes in Fortran 77 (Cambridge U. P.)

Sandage, A., Tammann, G. A., \& Reindl, B. 2004, A\&A, 424, 43

Schultheis, M., Ganesh, S., Simin, G., et al. 1999, A\&A, 349, L69

Stellingwerf, R. F. 1978, ApJ, 224, 953

Sumi, T. 2004, MNRAS, 349, 193

Udalski, A., Kubiak, M., Szymański, M., et al. 1994, AcA, 44, 317

Udalski, A., Szymański, M., Kaluzny, J., et al. 1995a, AcA, 45, 1

Udalski, A., Olech, A., Szymański, M., et al. 1995b, AcA, 45, 433

Udalski, A., Olech, A., Szymański, M., et al. 1996, AcA, 46, 51

Udalski, A., Olech, A., Szymański, M., et al. 1997, AcA, 47, 1

Udalski, A., Soszyński, I., Szymański, M., et al. 1998, AcA, 48, 563

Udalski, A., Szymański, M., Kubiak, M., et al. 2002, AcA, 52, 217

Wozniak, P. R., Udalski, A., Szymanski, M., et al. 2002, AcA, 52, 129

Wyrzykowski, L., Udalski, A., Kubiak, M., et al. 2003, AcA, 53, 1

Wyrzykowski, L., Udalski, A., Kubiak, M., et al. 2004, AcA, 54, 1

Wyithe, J. S. B., \& Wilson, R. E. 2001, ApJ, 559, 260

Zebruń, K., Soszyński, I., Woźniak, P., et al. 2001, AcA, 51, 317 